\title{
On lattice structure and implications on ordered fuzzy numbers
}

\author{
Magdalena Kacprzak ${ }^{1}$ Witold Kosiński ${ }^{1,2}$ \\ 1 Polish-Japanese Institute of Information Technology, ul. Koszykowa 86, 02-008 Warsaw, Poland \\ ${ }^{2}$ Kazimierz Wielki University of Bydgoszcz, 85-064 Bydgoszcz, Poland
}

\begin{abstract}
Ordered fuzzy numbers (OFN) invented by the second author and his two coworkers in 2002 make possible to utilize the fuzzy arithmetic and to construct the Abelian group of fuzzy numbers and then an ordered ring. The definition of OFN uses the extension of the parametric representation of convex fuzzy numbers. Fuzzy implication is proposed with the help of algebraic operations and a lattice structure defined on OFN.
\end{abstract}

Keywords: Convex fuzzy numbers, ordered fuzzy numbers, partial order, lattice, implication, defuzzification functionals.

\section{Introduction}

A fuzzy implication (FI), commonly defined as a two-place operation on the unit interval, is an extension of the classical binary implication. It plays important roles in both mathematical and applied sides of fuzzy set theory. The importance of fuzzy implications in applications of fuzzy logic (FL) to approximate reasoning (AR), decision support systems (DSS), fuzzy control (FC), etc., is hard to exaggerate. Many different fuzzy implication operators have been proposed; most of them fit into one of the two classes: implication operations that are based on an explicit representation of implication $A \Rightarrow B$ in terms of alternative, conjunction and negation and $R$-implications that are based on an implicit representation of implication $A \Rightarrow B$ as the weakest $C$ for which $C \wedge B$ implies $A$. However, some fuzzy implication operations cannot be naturally represented in this form [1].

For example, to the first class belong the KleeneDienes operation, called a binary implication, which is a fuzzy counterpart of the RHS of the binary logic tautology $a \Rightarrow b \equiv b \vee \neg a$. To have it one has to invent the negation operator $\neg$ to the membership function $\mu_{A}$ of a fuzzy set $A$ and to define a membership function of $\neg A$ as $\mu_{\neg A}:=1-\mu_{A}$, and the alternative of two fuzzy sets $C=A \vee B$, and its membership function $\mu_{C}$ as $\mu_{C}:=\max \left\{\mu_{A}, \mu_{B}\right\}$. Then the Kleene-Dienes implication $A \Rightarrow B$ will be $\max \left\{1-\mu_{A}, \mu_{B}\right\}$. The simple generalization of the last implication is the so-called $S$-implication
$I_{S}(A, B)$ defined by the formula

$$
I_{S}(A, B)=S\left(1-\mu_{A}, \mu_{B}\right)
$$

where $S$ is any $S$-norm [3]. This generalization is obvious in view of the fact that any $S$-norm is a generalization of the sum (alternative) of two fuzzy sets. Implication invented by Łukasiewicz [18] which takes the form $\min \left\{1,1-\mu_{A}+\mu_{B}\right\}$ together with the Reichenbach and Fodor implications serve as examples of $I_{S}(A, B)$ implication $[1,23]$.

The general class of the so-called $Q$-implication $I_{Q}(A, B)$ can be derived from the formula

$$
I_{Q}(A, B)=S\left(1-\mu_{A}, T\left(\mu_{A}, \mu_{B}\right)\right)
$$

where $S$ and $T$ are general $S$-norm and $T$-norm, respectively. The example of $Q$-implication is the Zadeh implication [26] defined by

$$
\max \left\{\min \left\{\mu_{A}, \mu_{B}\right\}, 1-\mu_{A}\right\} .
$$

The $R$-implication $I_{R}(A, B)$ is defined with the help of the formula

$$
I_{R}(A, B)=\sup \left\{z \in[0,1] \mid T\left(\mu_{A}, z\right) \leq \mu_{B}\right\} .
$$

Examples of $I_{R}(A, B)$ are the Gougen and Gödel implications [1].

It is no difficult to check that all above listed implications are consistent with the classical binary logic implication. There are, however, the socalled 'engineering' implications $M(A, B)$ invented by Mamdani, in which any $T$-norm appears, i.e.

$$
M(A, B)=T\left(\mu_{A}, \mu_{B}\right) .
$$

In the classical case [19] Mamdani put $T=\min$, then Larsen [16] proposed $T=$ prod, where prod represents the algebraic product; of course non of them is consistent with the classical binary implication.

If we define above implications on the set of convex fuzzy numbers (CFN) (cf. [20, 4]) then in most case the results of their operations lead outside the set OFN. Moreover, acting on convex fuzzy numbers of compact supports all those implications result with fuzzy sets, in general, of unbounded supports [21]. 


\section{Ordered fuzzy numbers}

Proposed recently by the second author and his two coworkers: Prokopowicz and Ślęzak [9, 10, 11, 12, 13] an extended model of convex fuzzy numbers [20] $(\mathrm{CFN})$, called ordered fuzzy numbers $(\mathrm{OFN})$, does not require any existence of membership functions.

Definition 1. An ordered fuzzy number is a pair $(f, g)$ of continuous functions defined on the interval $[0,1]$ with values in $\mathbf{R}$.

To see OFN as an extension of CFN - model, take a look on a parametric representation know since 1986, [4] of convex fuzzy numbers. In general, the functions $f, g$ need not to be invertible, as functions of $y \in[0,1]$, only continuity is required. If we assume that

1. $f$ is increasing, and $g$ is decreasing, and such that

2. $f \leq g$ (pointwise),

we might define the membership function

$$
\mu(x)= \begin{cases}f^{-1}(x), & \text { if } x \in[f(0), f(1)]=\left[l_{A}, 1_{A}^{+}\right], \\ g^{-1}(x), & \text { if } x \in[g(1), g(0)]=\left[1_{A}^{-}, p_{A}\right], \\ 1, & \text { if } x \in[f(1), g(1)]=\left[1_{A}^{+}, 1_{A}^{-}\right] .\end{cases}
$$

In this way obtained membership function $\mu(x), x \in \mathbf{R}$ represents a mathematical object which reminds a convex fuzzy number in the classical sense $[6,24]$. Notice that for the representation of the membership function $\mu$ of the convex fuzzy number one can attach two monotonic functions $\mu_{u p}:=f^{-1}$ and $\mu_{\text {down }}:=g^{-1}$ defined on the corresponding intervals $[f(0), f(1)]$ and $[g(1), g(0)]$, respectively. To be in agreement with further and classical denotations of fuzzy sets (numbers), the independent variable of the both functions $f$ and $g$ is denoted by $y$ (or some times by $s$ ), and their values by $x$. The continuity of both parts implies their images are bounded intervals, say $U P$ and $D O W N$, respectively, where boundaries for $U P=\left[l_{A}, 1_{A}^{+}\right]$, for $D O W N=\left[1_{A}^{-}, p_{A}\right]$ and for the CONST $=\left[1_{A}^{+}, 1_{A}^{-}\right]$ are used (see Fig. 1). Moreover, it may happen that the membership function corresponding to $A$ does not exist; such numbers are called improper.

Then four algebraic operations have been proposed between fuzzy numbers and crisp (real) numbers, in which componentwise operations are present. In particular if $A=\left(f_{A}, g_{A}\right), B=\left(f_{B}, g_{B}\right)$ and $C=\left(f_{C}, g_{C}\right)$ are mathematical objects called ordered fuzzy numbers, then the sum $C=A+B$, product $C=A \cdot B$, division $C=A \div B$ and scalar multiplication by real $r \in \mathbf{R}$, are defined in natural way:

$$
r \cdot A=\left(r f_{A}, r g_{A}\right),
$$

and for any $y \in[0,1]$

$$
f_{C}(y)=f_{A}(y) \star f_{B}(y), \quad g_{C}(y)=g_{A}(y) \star g_{B}(y),
$$
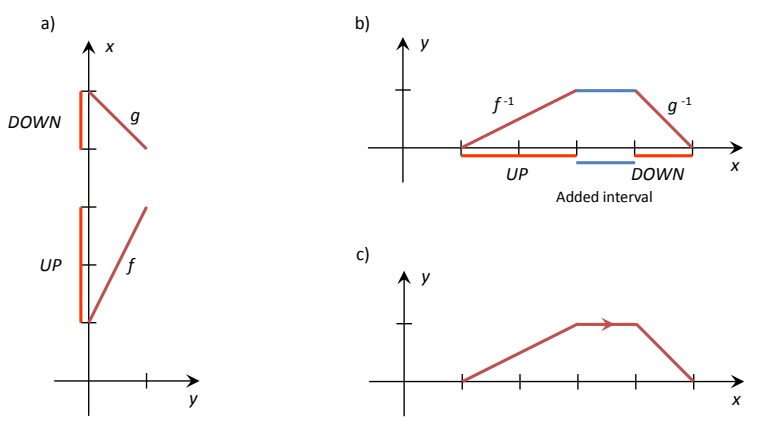

Figure 1: a) Example of an ordered fuzzy number; b) construction of the membership function; c) the arrow denotes the orientation and the order of inverted functions: first $f$ and then $g$.

where " $\star$ " works for " + ", ".", and " $\div$ ", respectively, and where $A \div B$ is defined, if the functions $\left|f_{B}\right|$ and $\left|g_{B}\right|$ are bigger than zero. Notice that the subtraction of $B$ is the same as the addition of the opposite of $B$, i.e. the number $(-1) \cdot B$, and consequently $B-B=0$. From this follows that any fuzzy algebraic equation $A+X=C$ with given $A$ and $C$ as OFN possesses a solution, that is OFN, as well. Moreover, to any convex and continuous ${ }^{11}$ fuzzy number correspond two OFNs, they differ by the orientation: one has positive, say $(f, g)$, another $(g, f)$ has negative. The space of all ordered fuzzy numbers will be denoted $\mathcal{R}$.

In the set $\mathcal{R}$ we intend to define new fuzzy implications. One of the most promising implications is that suggested by Prokopowicz in his Ph.D. thesis and called implication with multiplication [22], in which the algebraic structure of operations on OFN has been used. Prokopowicz's implication $A \Rightarrow B$, with $A$ and $B$ as two OFN has its value $v(A \Rightarrow B)$ given by

$$
v(A \Rightarrow B)=\mu_{A} B
$$

where the RHS of (7) is function of $x \in \operatorname{Range}(A)$ and $s \in I$, and is valid for the case when $A$ is proper $^{2}$, i.e., it possesses the membership function $\mu_{A}$, given by (5). If the premise part in given by the improper ordered fuzzy number $A$, when the formula (5) cannot be used, a new, co-called corresponding membership function $\tilde{\mu}_{A}$ can be defined $[22]$ as

$\tilde{\mu}_{A}(x)= \begin{cases}\max \arg \{f(s)=x, g(y)=x\}, & \text { if } x \in \mathcal{A}_{1}, \\ 1, & \text { if } x \in \mathcal{A}_{2}, \\ 0, & \text { otherwise }\end{cases}$

where $\mathcal{A}_{1}=\operatorname{Range}(f) \cup \operatorname{Range}(g)$, and $\mathcal{A}_{2}=$ $[f(1), g(1)] \cup[g(1), f(1)], s \in I=[0,1]$, and one

\footnotetext{
${ }^{11}$ However, the recent extension presented in [8] includes all convex fuzzy numbers.

${ }^{2}$ If $f$ is strictly increasing and $g$ decreasing, and $f(s) \leq$ $g(s)$, for $s \in[0,1]$, then $A$ is called proper and it possesses the classical membership function, moreover it represents a CFN.
} 
of the intervals $[f(1), g(1)]$ or $[g(1), f(1)]$ may be empty, depending on the sign of $f(1)-g(1)$, (i.e., if the sign is -1 then the second interval is empty). Then in the RHS of 7 ) we put $\tilde{\mu}_{A}$ instead of $\mu_{A}$. The multiplication on the right hand side is defined with the use of (6), i.e. if $v(A \Rightarrow B)(x, s)=\mu_{A}(x) B(s)$, for $s \in I$. The result of this implication is an ordered fuzzy number and is close to the engineering implication of Mamdani type, rather.

In dealing with applications of fuzzy numbers we need the set of functionals that map each fuzzy number into real, and in such a way that is consistent with operations on reals. Those operations are called defuzzifications. To be more strict we introduce

Definition 2. A map $\phi$ from the space of all OFN's to reals is called a defuzzification functional if it satisfies:

1. $\phi\left(c^{\ddagger}\right)=c$,

2. $\phi\left(A+c^{\ddagger}\right)=\phi(A)+c$,

3. $\phi(c A)=c \phi(A)$, for any $c \in \mathbf{R}$ and $A \in \mathcal{R}$

where $c^{\ddagger}(s)=(c, c), s \in[0,1]$, represents crisp number (a real) $c \in \mathbf{R}$.

From this follow that each defuzzification functional must be homogeneous of order one, restrictive additive, and some how normalized.

\subsection{Step ordered fuzzy numbers}

It is worthwhile to point out that a class of ordered fuzzy numbers (OFNs) represents the whole class of convex fuzzy numbers with continuous membership functions. To include all CFN some generalization of functions $f$ and $g$ in Def. 1 is needed. This has been already done by the second author who in [8] assumed they are functions of bounded variation. Then operations are defined in the similar way, the norm, however, will change into the norm of the cartesian product of the space of functions of bounded variations (BV). Then all convex fuzzy numbers are contained in this new space $\mathcal{R}_{B V}$, of OFN. Notice that functions from BV [17] are continuous except for a countable numbers of points.

Important consequence of this generalization is a possibility of introducing a subspace of OFN composed of pairs of step functions ([14]). If we fix a natural number $K$ and split $[0,1)$ into $K-1$ subintervals $\left[a_{i}, a_{i+1}\right)$, i.e. $\bigcup_{i=1}^{K-1}\left[a_{i}, a_{i+1}\right)=[0,1)$, where $0=a_{1}<a_{2}<\ldots<a_{K}=1$, and define a step function $f$ of resolution $K$ by putting value $u_{i}$ on each subinterval $\left[a_{i}, a_{i+1}\right)$, then each such function $f$ is identified with a $K$-dimensional vector, i.e., $f \sim \boldsymbol{u}=\left(u_{1}, u_{2} \ldots u_{K}\right) \in \mathbf{R}^{K}$, the $K$-th value $u_{K}$ corresponds to $y=1$, i.e. $f(1)=u_{K}$. Taking a pair of such functions we have an ordered fuzzy number from $\mathcal{R}_{B V}$. Now we introduce

Definition 3. By a step ordered fuzzy number $A$ of

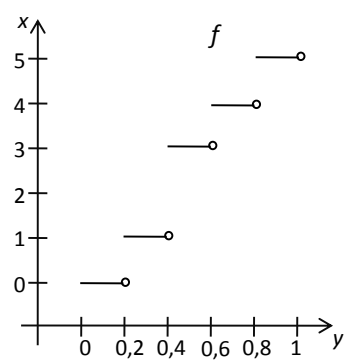

(a)

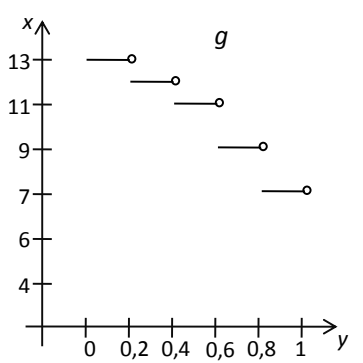

(b)
Figure 2: Example of a step ordered fuzzy number $A=(f, g) \in \mathcal{R}_{K}$, (a) function $f$, (b) function $g$.

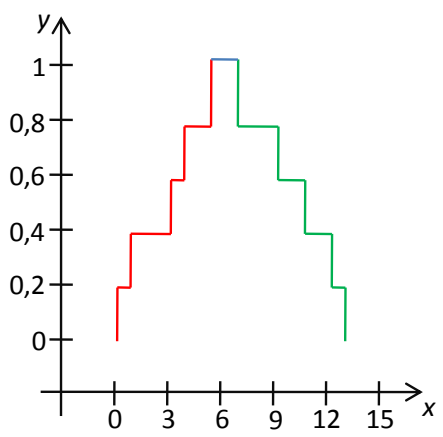

Figure 3: Membership relation of the above step ordered fuzzy number $A=(f, g) \in \mathcal{R}_{K}$.

resolution $K$ we mean an ordered pair $(f, g)$ of functions such that $f, g:[0,1] \rightarrow \mathbf{R}$ are step functions of resolution $K$.

We use $\mathcal{R}_{K}$ for denotation the set of elements satisfying Def. 3. The example of a step ordered fuzzy number and its membership relation (represented by a curve) are shown in Fig. 2 and Fig. 3. The set $\mathcal{R}_{K} \subset \mathcal{R}_{B V}$ has been extensively elaborated by our students in [5] and [15]. We can identify $\mathcal{R}_{K}$ with the Cartesian product of $\mathbf{R}^{K} \times \mathbf{R}^{K}$ since each $K$-step function is represented by its $K$ values. It is obvious that each element of the space $\mathcal{R}_{K}$ may be regarded as an approximation of elements from $\mathcal{R}_{B V}$, by increasing the number $K$ of steps we are getting the better approximation. The norm of $\mathcal{R}_{K}$ is assumed to be the Euclidean one of $\mathbf{R}^{2 K}$, then we have a inner-product structure for our disposal.

\subsection{Defuzzification functionals on $\mathcal{R}_{K}$}

On the space $\mathcal{R}_{K}$ a representation formula for a general non-linear defuzzification functional $H: \mathbf{R}^{K} \times \mathbf{R}^{K} \rightarrow \mathbf{R}$ satisfying the conditions 1.- 3 . of Def.2, can be given as a linear composition [14] of arbitrary homogeneous of order one, continuous function $G$ of $2 K-1$ variables, with the 1D identity 
function, i.e.

$$
\begin{array}{r}
H(\underline{u}, \underline{v})=u_{j}+ \\
G\left(u_{2}-u_{j}, \ldots, u_{K}-u_{j}, v_{1}-u_{j}, \ldots, v_{K}-u_{j}\right), \\
\text { with } \underline{u}=\left(u_{1}, \ldots, u_{K}\right), \underline{v}=\left(v_{1}, \ldots, v_{K}\right),
\end{array}
$$

and some $1 \leq j \leq K$. It is seen that $G$ is given by $F$ in which its $j$-th argument was put equal to zero.

Due to the fact that $\mathcal{R}_{K}$ is isomorphic to $\mathbf{R}^{K} \times$ $\mathbf{R}^{K}$ we conclude, from the Riesz theorem and the condition 1. that a general linear defuzzification functional on $\mathcal{R}_{K}$ has the representation

$$
H(\underline{u}, \underline{v})=\underline{u} \cdot \underline{b}+\underline{v} \cdot \underline{d},
$$

with arbitrary $\underline{b}, \underline{d} \in \mathbf{R}^{K}$, such that $\underline{1} \cdot \underline{b}+\underline{1} \cdot \underline{d}=1$, where $\cdot$ denotes the inner (scalar) product in $\mathbf{R}^{K}$ and $\underline{1}=(1,1, \ldots, 1) \in \mathbf{R}^{K}$ is the unit vector in $\mathbf{R}^{K}$, while the pair $(\underline{1}, \underline{1})$ represents a crisp one in $\mathcal{R}_{K}$. It means that such a functional is represented by the vector $(\underline{b}, \underline{d}) \in \mathbf{R}^{2 K}$. Notice that all functionals of the type $\phi_{j}=\underline{e}_{j}, j=1,2, \ldots, 2 K$, where $\underline{e}_{j} \in$ $\mathbf{R}^{2 K}$ has all zero component except for 1 on the $j$ th position, form a basis of $\mathcal{R}_{K}{ }^{*}$ - the space adjoint to $\mathcal{R}_{K}$, they are called fundamental functionals .

Let us take $\underline{b}=\underline{c}$ and such, that all their components are equal to $1 / 2 K$, and denote such defuzzification functional by $\psi_{K}$.

Now let us introduce the particular subset $\mathcal{N}$ of $\mathcal{R}_{K}$, with $\underline{u}, \underline{v} \in \mathcal{N}$ such that each component of the vector $\underline{u}$ as well as of $\underline{v}$ has value 1 or 0 . Since each element of $\mathcal{N}$ is represented by a $2 K$-dimensional binary vector the cardinality of the set $\mathcal{N}$ is $2^{2 K}$. Then if we apply the functional $\psi_{K}$ to elements of $\mathcal{N}$ we may obtain all possible fractional numbers $i / 2 K$, with $i=0,1, \ldots, 2 K$, as the values of defuzzification functional $\psi_{K}$ on $\mathcal{N}$. The set $\mathcal{N}$ and the functional $\psi_{K}$ will play fundamental roles in the next sections.

\section{Lattice structure on ordered fuzzy numbers}

In this section we define a partial order on the set of all ordered fuzzy numbers and we introduce two binary operations: conjunction and disjunction, in order to joint two ordered fuzzy numbers. Next we show that OFN with these operations creates a lattice.

\subsection{Partial order on OFN}

A relation of partial ordering in the space $\mathcal{R}$ of all OFN can be introduced by defining the subset of 'positive' ordered fuzzy numbers: a number $A=$ $(f, g)$ is not less than zero, and by writing

$$
A \geq 0 \text { iff } f \geq 0, g \geq 0 .
$$

In this way the set $\mathcal{R}$ becomes a partially ordered ring if we define the partial order relation $\geq$ between elements of $\mathcal{R}$ as

$$
A \leq B \quad \text { iff } \quad B-A \geq 0 .
$$

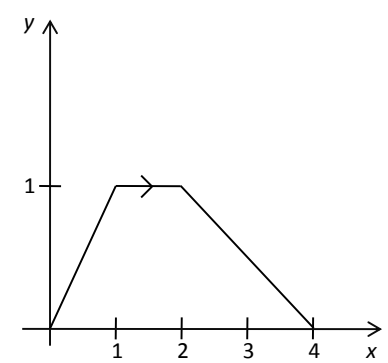

(a)

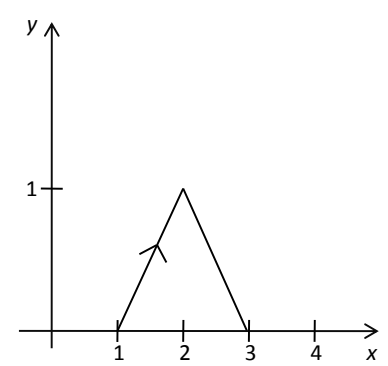

(b)
Figure 4: Ordered fuzzy numbers (a) $A=(y, 4-2 y)$, (b) $C=(1+y, 3-y)$.

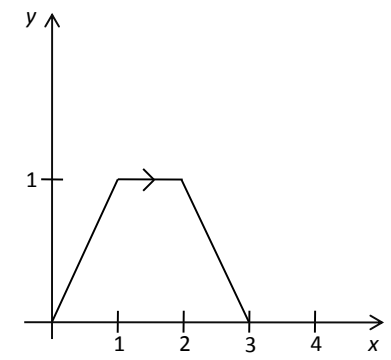

(a)

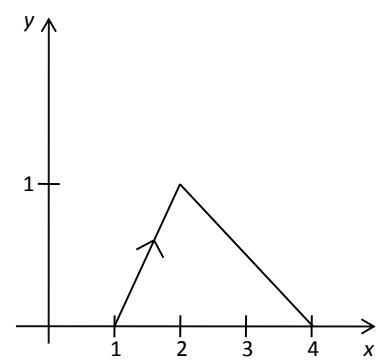

(b)
Figure 5: Conjunction and disjunction of the above fuzzy numbers: (a) $A \wedge C=(y, 3-y)$, (b) $A \vee C=$ $(y+1,4-2 y)$.

To show an example assume that $A=(y, 4-2 y)$ and $B=(y+1,4-y)$. Then $A \leq B$ since $B-A=(1, y)$ does not achieve negative values for $y \in[0,1]$. At the same time ordered fuzzy numbers $A=(y, 4-2 y)$ and $C=(y+1,3-y)$ are not comparable since neither $A-C \geq 0$ nor $C-A \geq 0$ for $y \in[0,1]$.

The space $\mathcal{R}$ becomes a Banach space (or even a Banach algebra with unity) if we equip it with the topology (and the norm) of the Cartesian product of $C([0,1]) \times C([0,1])$, where $C([0,1])$ is the space of continuous functions defined on $[0,1]$ with the sup (Chebyshev) norm.

\subsection{Conjunction and disjunction connectives defined on OFN}

By analogy to classical logic, for each two ordered fuzzy numbers we define operations of conjunction, noted by $\wedge$, and disjunction, noted by $\vee$. Let $A=$ $\left(f_{A}, g_{A}\right), B=\left(f_{B}, g_{B}\right) \in \mathcal{R}$ then

$$
A \wedge B=\left(\inf \left\{f_{A}, f_{B}\right\}, \inf \left\{g_{A}, g_{B}\right\}\right)
$$

and

$$
A \vee B=\left(\sup \left\{f_{A}, f_{B}\right\}, \sup \left\{g_{A}, g_{B}\right\}\right) .
$$

For example if $A=(y, 4-2 y)$ and $B=(y+1,4-y)$, then $A \wedge B=(y, 4-2 y)=A$ and $A \vee B=(y+1,4-$ $y)=B$. For ordered fuzzy numbers $A=(y, 4-2 y)$ and $C=(y+1,3-y)$ depicted in Fig.4 conjunction 
and disjunction connectives are calculated as follows $A \wedge C=(y, 3-y)$ and $A \vee C=(y+1,4-2 y)$ - see Fig.5.

The operations will be used in the definition of the lattice structure on OFN.

\subsection{Lattice on $\mathcal{R}$}

Let us consider the set $\mathcal{R}$ of ordered fuzzy numbers with operations $\vee$ and $\wedge$. Notice that $\vee$ and $\wedge$ are operations in $\mathcal{R}$, i.e. are defined for all $A, B \in$ $\mathcal{R}$ and the result of the operations is in $\mathcal{R}$. Next, observe that operation $\vee$ is

- idempotent i.e. whenever it is applied to two equal values, it gives that value as the result:

$$
\begin{gathered}
A \vee A=\left(\sup \left\{f_{A}, f_{A}\right\}, \sup \left\{g_{A}, g_{A}\right\}\right)= \\
\left(f_{A}, g_{A}\right)=A
\end{gathered}
$$

- commutative:

$$
\begin{gathered}
A \vee B=\left(\sup \left\{f_{A}, f_{B}\right\}, \sup \left\{g_{A}, g_{B}\right\}\right)= \\
\left(\sup \left\{f_{B}, f_{A}\right\}, \sup \left\{g_{B}, g_{A}\right\}\right)=B \vee A
\end{gathered}
$$

- associative:

$$
\begin{gathered}
(A \vee B) \vee C=\left(\sup \left\{f_{A}, f_{B}\right\}, \sup \left\{g_{A}, g_{B}\right\}\right) \vee C= \\
\left(\sup \left\{f_{A}, f_{B}, f_{C}\right\}, \sup \left\{g_{A}, g_{B}, g_{C}\right\}\right)= \\
A \vee\left(\sup \left\{f_{B}, f_{C}\right\}, \sup \left\{g_{B}, g_{C}\right\}\right)=A \vee(B \vee C)
\end{gathered}
$$

The same properties has the operation $\wedge$. Moreover, these two operations are connected by the absorption law:

$$
\begin{gathered}
A \wedge(A \vee B)=A \wedge\left(\sup \left\{f_{A}, f_{B}\right\}, \sup \left\{g_{A}, g_{B}\right\}\right)= \\
\left(\inf \left\{f_{A}, \sup \left\{f_{A}, f_{B}\right\}\right\}, \inf \left\{g_{A}, \sup \left\{g_{A}, g_{B}\right\}\right\}\right)= \\
\left(f_{A}, g_{A}\right)=A
\end{gathered}
$$

and similar for

$$
A \vee(A \wedge B)=A \text {. }
$$

The absorption laws ensure that the set $\mathcal{R}$ with an order $\leq$ defined as

$$
A \leq B \quad \text { iff } \quad B=A \vee B
$$

is a partial ordering within which meets and joins are given through the operations $\vee$ and $\wedge$. It is easy to show that for every $A, B \in \mathcal{R}$ it holds that $A \vee B=B$ iff $B-A \geq 0$. It means that the orders defined by (12) and (15) are equivalent. Moreover, joints and meets exist for every two elements of $\mathcal{R}$. The following theorem is the consequence of the above reasoning.

Theorem 1. The algebra $(\mathcal{R}, \vee, \wedge)$ is a lattice.

\subsection{Compliments and lattice on $\mathcal{N}$}

Now consider the subset $\mathcal{N}$ of $\mathcal{R}_{K}$ defined in Section 2.2. As we said before every element of $\mathcal{N}$ can be represented by a binary vector and thereby $\mathcal{N}$ is isomorphic to the space of Boolean vectors. Below,

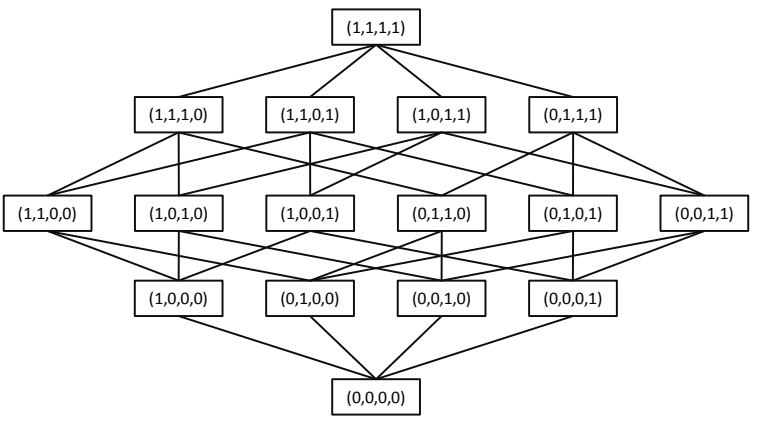

Figure 6: A complete and complemented lattice defined on a set of step ordered fuzzy numbers $\mathcal{N} \subset \mathbf{R}^{4}$.

we use the notation $A_{\left(a_{1}, a_{2}, \ldots a_{2 K}\right)}$ for a number $A$ represented by vector $\left(a_{1}, a_{2}, \ldots a_{2 K}\right)$ and we show that $\mathcal{N}$ is a Boolean algebra.

It is easy to observe that all subsets of $\mathcal{N}$ have both a join and a meet in $\mathcal{N}$. In fact, for every pair of numbers from the set $\{0,1\}$ we can determine $\max$ and $\min$ and it is always 0 or 1 . Therefore $\mathcal{N}$ creates a complete lattice. In such a lattice we can distinguish the greatest element $\underline{1}=A_{(1,1, \ldots, 1)}$ and the least element $\underline{0}=A_{(0,0, \ldots, 0)}$.

Theorem 2. The algebra $(\mathcal{N}, \vee, \wedge)$ is a complete lattice.

In a lattice in which the greatest and the least elements exist it is possible to define compliments. We say that two elements $A$ and $B$ are complements of each other if and only if

$$
A \vee B=\underline{1} \text { and } A \wedge B=\underline{0} .
$$

The compliment of a number $A$ will be marked with $\neg A$ and is defined as follows:

Definition 3. Let $A_{\left(a_{1}, a_{2}, \ldots, a_{2 K}\right)} \in \mathcal{N}$ be a step ordered fuzzy number. Then the compliment of $A_{\left(a_{1}, a_{2}, \ldots, a_{2 K}\right)}$ equals

$$
\neg A_{\left(a_{1}, a_{2}, \ldots, a_{2 K}\right)}=A_{\left(1-a_{1}, 1-a_{2}, \ldots, 1-a_{2 K}\right)} .
$$

A bounded lattice for which every element has a complement is called a complemented lattice. The structure of step ordered fuzzy numbers $(\mathcal{N}, \vee, \wedge)$ forms complete and complemented lattices in which complements are unique. In fact it is a Boolean algebra. An example of such an algebra is depicted in Fig. 6. A set of universe is created by numbers $\mathcal{N}=$ $\left\{A_{\left(a_{1}, a_{2}, a_{3}, a_{4}\right)}: a_{i} \in\{0,1\}\right.$ for $\left.i=1,2,3,4\right\}$. The compliments of elements are $\neg A_{(0,0,0,0)}=A_{(1,1,1,1)}$, $\neg A_{(0,1,0,0)}=A_{(1,0,1,1)}, \neg A_{(1,1,0,0)}=A_{(0,0,1,1)}$ etc.

\section{New fuzzy implication}

Our aim is to propose an operation of implication on ordered fuzzy numbers analogous to classical implication and which preserves its main properties. 


\begin{tabular}{|c|c|c|}
\hline$A$ & $B$ & $A \rightarrow B$ \\
\hline \hline$A_{(0,0,1,0)}$ & $A_{(1,1,0,1)}$ & $A_{(1,1,0,1)}$ \\
\hline$A_{(0,0,1,0)}$ & $A_{(1,0,0,0)}$ & $A_{(1,1,0,1)}$ \\
\hline$A_{(0,0,1,1)}$ & $A_{(0,0,1,0)}$ & $A_{(1,1,1,0)}$ \\
\hline$A_{(1,0,0,1)}$ & $A_{(0,1,0,0)}$ & $A_{(0,1,1,0)}$ \\
\hline$A_{(1,1,0,0)}$ & $A_{(1,1,0,0)}$ & $A_{(1,1,1,1)}$ \\
\hline$A_{(1,1,1,1)}$ & $A_{(1,0,0,0)}$ & $A_{(1,0,0,0)}$ \\
\hline
\end{tabular}

Table 1: Examples of implications for step ordered fuzzy numbers.

One of them says that for any two formulas $\alpha$ and $\beta$, formula if $\alpha$ then $\beta$, i.e. $\alpha \Rightarrow \beta$ is equivalent to formula $\neg \alpha \vee \beta$. Similar equality we want to obtain for OFN. In Section 3.2 we introduced a definition of disjunction operation for any two order fuzzy numbers. The problem is with operation of negation. In Section 3.4 we showed how compliments for step ordered fuzzy numbers from the set $\mathcal{N}$ are constructed. So, given disjunction and compliment, implication can be defined in the standard way. Since the set of all ordered fuzzy numbers is not a complete lattice, the way of defining of implication is not straightforward. Below, we propose new binary operator on the set $\mathcal{N}$ which is called $2 K$-fuzzy implication. It is an open question how to define negation and implication on the set of all ordered fuzzy numbers $\mathcal{R}$.

Assume a Boolean algebra $(\mathcal{N}, \vee, \wedge, \neg, \underline{1}, \underline{0})$. We introduce

Definition 4 For any $A, B \in \mathcal{N}$ the operation of $2 \mathrm{~K}$-fuzzy implication $\rightarrow$ is defined as

$$
A \rightarrow B:=\neg A \vee B .
$$

In other words, the result of binary operation $A \rightarrow B$ is equal to the result of operation sup for the number $B$ and the compliment of $A$ :

$$
A \rightarrow B=\sup \{\neg A, B\} .
$$

For illustration, assume two numbers $A_{(0,1,1,0)}$ and $A_{(0,1,0,1)}$. The implication

$$
A_{(0,1,1,0)} \rightarrow A_{(0,1,0,1)}
$$

equals

$$
\begin{gathered}
\neg A_{(0,1,1,0)} \vee A_{(0,1,0,1)}= \\
A_{(1,0,0,1)} \vee A_{(0,1,0,1)}=A_{(1,1,0,1)} .
\end{gathered}
$$

Examples of other implications are given in Table 1 $2 \mathrm{~K}$-fuzzy implication satisfies the basic property of the logical implication, i.e., it returns false if and only if the first term is true, and the second term is false.

Proposition 1 Consider the Boolean algebra $(\mathcal{N}, \vee, \wedge, \neg, \underline{1}, \underline{0})$. The values of the $2 K$-fuzzy implication on the

\begin{tabular}{|c|c|c|}
\hline$A$ & $B$ & $A \rightarrow B$ \\
\hline \hline$\underline{0}$ & $\underline{0}$ & $\underline{1}$ \\
\hline$\underline{0}$ & $\underline{1}$ & $\underline{1}$ \\
\hline$\underline{1}$ & $\underline{0}$ & $\underline{0}$ \\
\hline$\underline{1}$ & $\underline{1}$ & $\underline{1}$ \\
\hline
\end{tabular}

Table 2: The table of values of implications for the least element and the greatest elements of $\mathcal{N}$.

greatest and the least elements of this algebra are given in the Table 2 .

In fact, since $\neg \underline{0}=\underline{1}$ and $\neg \underline{1}=\underline{0}$ it holds that:

- $\underline{0} \rightarrow \underline{0}=\neg \underline{0} \vee \underline{0}=\underline{1} \vee \underline{0}=\underline{1}$

- $\underline{0} \rightarrow \underline{1}=\neg \underline{0} \vee \underline{1}=\underline{1} \vee \underline{1}=\underline{1}$

- $\underline{1} \rightarrow \underline{0}=\neg \underline{1} \vee \underline{0}=\underline{0} \vee \underline{0}=\underline{0}$

- $\underline{1} \rightarrow \underline{1}=\neg \underline{1} \vee \underline{1}=\underline{0} \vee \underline{1}=\underline{1}$

Having the described above implication we can apply step ordered fuzzy numbers for evaluation of linguistic statements like "a patient is fat" or "a car is fast" and reasoning on them. Below, we present a method of assigning elements of the set $\mathcal{N}$ to ordered fuzzy numbers representing such statements. Notice that some how our $2 K$-implication satisfies conditions of fuzzy implication, classically formulated in the theory of fuzzy sets [1] for two-value operations on a complete lattice (in particular case on the interval $[0,1]$ ) requiring from it to be decreasing with respect to the first variable, increasing with respect to the second variable and fulfilling three boundary conditions corresponding to 1,2 and 3 in the rows of Table above.

Consider a classical (convex) fuzzy number $Z \in$ CFN with its membership function $\mu_{Z}$. Let us recall that for $Z$ we may define for each $s \in(0,1]$ the $s$ cut (or $s$-section) of the number (of the membership function) $Z$ as the classical set $Z_{s}$ by

$$
Z_{s}=\left\{x \in \mathbf{R}: \mu_{Z}(x) \geq s\right\} .
$$

For each convex fuzzy number $Z$ and two numbers $s_{1} \leq s_{s}$ the following relation $Z_{s_{2}} \subset Z_{s_{1}}$ between the corresponding $s$-sections holds.

\subsection{Possible application}

Now let us fix the resolution $K$ of step functions defining the $\mathcal{R}_{K}$ and take the partition of the unit interval into $K-1$ subintervals $\bigcup_{i=1}^{K-1}\left[a_{i}, a_{i+1}\right) \cup$ $\left\{a_{K}\right\}=[0,1]$, with $0=a_{1}<a_{2}<\ldots<a_{K}=1$.

Then we may define a mapping

$$
\mathrm{val}_{K}: \mathbf{R} \times \mathrm{CFN} \rightarrow \mathcal{N}
$$

which for given $Z$ and each $x \in Z_{a_{i}}-Z_{a_{i+1}}$ attaches an element of the set $\mathcal{N}$, a step ordered fuzzy number, in such a way that $\psi_{K}\left(\operatorname{val}_{K}(x, Z)\right)=a_{i}$, i.e. after defuzzifying the value of $\operatorname{val}_{K}(x, Z)$ we get the 
value of the membership function of $Z$ at the lower end of the $s$-section to which $x$ belongs. In this way the one-variable function $\operatorname{val}_{K}(\cdot, Z): \mathbf{R} \rightarrow \mathcal{N}$ is piecewise constant: it is constant on each subinterval $Z_{a_{i}}-Z_{a_{i+1}}$. It means that after defuzzification the correspondence given by the function $\operatorname{val}_{K}(\cdot, Z)$ is in agreement with the value of the membership function attached to $x$ by $\mu_{Z}$, module the assumed finite step-wise approximation of values of the membership function.

If we use the so-called parametric representation of convex fuzzy numbers [4] in terms of two leftcontinuous functions $\alpha_{1}, \alpha_{2}$, the both defined on the interval $[0,1]$ with values in $\mathbf{R}$, and denote by $x_{1^{-}}$ and $x_{1}+$ the points from the support of $\mu_{Z}$, such that $\mu_{Z}\left(x_{1^{-}}\right)=\mu_{Z}\left(x_{1^{+}}\right)=1$, and at the point $x_{1^{-}}$ the membership function attains for the first time the value 1 , and the point $x_{1}+$ is the last point with this property ${ }^{3}$, then the condition $x \in Z_{a_{i}}-Z_{a_{i+1}}$, may be written as $\alpha_{1}\left(a_{i}\right) \leq x \leq \alpha_{2}\left(a_{i+1}\right)$ if $x \leq x_{1^{-}}$ and $\alpha_{1}\left(a_{i+1}\right) \leq x \leq \alpha_{2}\left(a_{i}\right)$ if $x \geq x_{1+}$. This is so, because the function $\alpha_{1}$ is non-decreasing and the function $\alpha_{2}$ is non-increasing.

Notice that for a classical fuzzy rule $[3,2,26]$ : If 'a condition is satisfied' Then 'a consequence follows', where both parts: premise and consequent are fuzzy, the mapping val $_{K}$ may be applied to the both and then our new fuzzy inference ${ }^{4}$ can be used.

\section{Conclusion}

So far, ordered fuzzy numbers was applied to deal with optimization problems when data are fuzzy. In this paper we present how they can be used for approximate reasoning about uncertainty linguistic propositions. In order to do this a new fuzzy implication on step ordered fuzzy numbers is introduced. In classical two-valued logics only two logical values are applied: 0 or 1 . In fuzzy logics it is extended to the values from the interval $[0,1]$. Our contribution is to enrich these formal systems and use for logical justification step ordered fuzzy numbers. This approach is very innovative and allows for including in logical value more information than that something is true, true with some degree or false. In future work we are going to show application and usefulness of this new reasoning on diverse examples, especially for modelling uncertain beliefs of agents in multi-agent systems.

Acknowledgement. The authors wishes to thank Dr. Baczyński for his suggestions concerning the lattice structure of OFN.

\section{References}

[1] Baczyński Michał and Jayaram Balasubramaniam (2008), Fuzzy Implications, Series: Stud-

\footnotetext{
${ }^{3}$ It means that $x_{1-}=\alpha_{1}(1)$ and $x_{1+}=\alpha_{2}(1)$.

${ }^{4}$ It seems that our approach reminds to some extend the fuzzy sets of type two introduced by Zadeh in [27] in 1975.
}

ies in Fuzziness and Soft Computing, vol. 231, Springer, Berlin 2008, ISBN: 978-3-540-69080-1,

[2] Buckley James J. (1992), Solving fuzzy equations in economics and finance, Fuzzy Sets and Systems, 48, 289-296.

[3] Czogała E., Pedrycz W. (1985), Elements and Methods of Fuzzy Set Theory (in Polish), PWN, Warszawa, Poland.

[4] Goetschel R. Jr., Voxman W. (1986), Elementary fuzzy calculus, Fuzzy Sets and Systems, 18 (1), 31-43.

[5] Gruszczyńska A., Krajewska I.,(2008). Fuzzy calculator on step ordered fuzzy numbers, in Polish, UKW, Bydgoszcz, 2008.

[6] Klir G.J., Fuzzy arithmetic with requisite constraints, Fuzzy Sets and Systems, 91 (1997) 165175.

[7] Kosiński W. (2004), On defuzzyfication of ordered fuzzy numbers, in: ICAISC 2004, 7th Int. Conference, Zakopane, Poland, June 2004, L. Rutkowski, Jörg Siekmann, Ryszard Tadeusiewicz, Lofti A. Zadeh (Eds.) LNAI, vol. 3070, pp. 326-331, Springer-Verlag, Berlin, Heidelberg, 2004.

[8] Kosiński W. (2006), On fuzzy number calculus, Int. J. Appl. Math. Comput. Sci., 16 (1), 51-57.

[9] Kosiński W., Prokopowicz P. , Ślęzak D. (2002), Fuzzy numbers with algebraic operations: algorithmic approach, in: Intelligent Information Systems 2002, M. Klopotek, S.T. Wierzchoń, M. Michalewicz(Eds.) Proc.IIS'2002, Sopot, June 3-6, 2002, Poland, pp. 311-320, Physica Verlag, Heidelberg, 2002.

[10] Kosiński W., Prokopowicz P., Ślȩzak D. (2002), Drawback of fuzzy arithmetics - new intutions and propositions, in: Proc. Methods of Aritificial Intelligence, T. Burczyński, W. Cholewa, W. Moczulski(Eds.), pp. 231-237, PACM, Gliwice, Poland, 2002.

[11] Kosiński W., P. Prokopowicz P., Ślȩzak D. (2003), On algebraic operations on fuzzy numbers, in Intelligent Information Processing and Web Mining, Proc. of the International IIS: IIPWM,03 Conference held in Zakopane, Poland, June 2-5,2003, M. Klopotek, S.T. Wierzchoń, K. Trojanowski(Eds.), pp. 353362, Physica Verlag, Heidelberg, 2003.

[12] Kosiński W., Prokopowicz P., Ślȩzak D.(2003), Ordered fuzzy numbers, Bulletin of the Polish Academy of Sciences, Sér. Sci. Math., 51 (3), 327-338.

[13] Kosiński W., Prokopowicz P. (2004), Algebra of fuzzy numbers (In Polish: Algebra liczb rozmytych), Matematyka Stosowana. Matematyka dla Spoleczeństwa, 5 (46), 37-63 .

[14] Kosiński W.,(2010) Optimization with fuzzy data via evolutionary algorithms, in ICNAAM 2010, International Conference on Numerical Analysis and Applied Mathematics 2010, Rhodes Greece, 19-25 September 2010, 2010 American 
Institute of Physics, CD-ROM ISBN978-0-735408-31-9

[15] Kościeński K. (2010), Moduł schodkowych liczb rozmytych $w$ sterowniu ruchem punktu materialnego, in Polish, PJIIT, Warszawa.

[16] Larsen P. M. (1980), Industrial applications of fuzzy logic controler, Intern. J. Man-Machine Studies, 12 (1), 3-10.

[17] Łojasiewicz S. (1973), Introduction to the Theory of Real Functions (in Polish), Biblioteka Matematyczna, Tom 46, PWN, Warszawa.

[18] Eukasiewicz J.(1958), Elements of the Mathematical Logic (in Polish), PWN, Warszawa, 1958.

[19] Mamdani, E.H. (1976), Advances in the linguistic synthesis of fuzzy controllers, Intern. J. Man-Machine Studies, 8, 669-678.

[20] Nguyen H.T. (1978), A note on the extension principle for fuzzy sets, J. Math. Anal. Appl.64, 369-380 .

[21] Piegat A.(1999), Fuzzy Modelling and Control, (In Polish: Modelowanie i sterowanie rozmyte), Akademicka Oficyna Wydawnicza PLJ, Warszawa.

[22] Prokopowicz P. (2005), Algorithmization of Operations on Fuzzy Numbers and its Applications (In Polish: Algorytmizacja działań na liczbach rozmytych i jej zastosowania), Ph. D. Thesis, IPPT PAN, kwiecień 2005.

[23] Rutkowski Leszek (2006), Metody i techniki sztucznej inteligencji - Inteligencja obliczeniowa, Wydawnictwo Naukowe PWN, Warszawa, 2006.

[24] Wagenknecht M., (2001), On the approximate treatment of fuzzy arithmetics by inclusion, linear regression and information content estimation, in: Zbiory rozmyte $i$ ich zastosowania, Fuzzy sets and their applications, J. Chojcan, J. Łȩski (eds.), Wydawnictwo Politechniki Śląskiej, Gliwice, 291-310.

[25] M. Wagenknecht, R. Hampel, V. Schneider, Computational aspects of fuzzy arithmetic based on Archimedean t-norms, Fuzzy Sets and Systems, 123/1 (2001) 49-62.

[26] Zadeh L. A.(1983), The role of fuzzy logic in the management of uncertainty in expert systems, Fuzzy Sets and Systems, 11(3), 199-227.

[27] Zadeh L.A., (1975). The concept of a linguistic variable and its application to approximate reasoning, Parts I-III, Information Sciences, 8, (8) 199-249, (8) 302-357, (9) 43-80. 